\title{
Impact of the malicious input data modification on the efficiency of quantum spatial search
}

\author{
Adam Glos ${ }^{1,2}$ (D) Jarosław Adam Miszczak ${ }^{1}$ (D)
}

Received: 11 April 2019 / Accepted: 17 September 2019 / Published online: 24 September 2019

(c) The Author(s) 2019

\begin{abstract}
In this paper, we demonstrate that the efficiency of quantum spatial search can be significantly altered by malicious manipulation of the input data in the client-server model. We achieve this by exploiting exceptional configuration effect on Szegedy spatial search and proposing a framework suitable for analysing efficiency of attacks on quantum search algorithms. We provide the analysis of proposed attacks for different models of random graphs. The obtained results demonstrate that quantum algorithms in general are not secure against input data alteration.
\end{abstract}

Keywords Quantum spatial search · Exceptional configurations · Cyberattacks . Quantum hacking

\section{Introduction}

\subsection{Motivation}

While the intensive research effort invested in the area of quantum computing is fully justified by groundbreaking theoretical developments [1-3], year by year scientists have discovered new limitations of quantum computing devices [4]. Quantum algorithms have been proved to be susceptible to noise, which may falsify the results of the computation. This fact motivated the development of the theory of quantum error-correcting codes. Unitary operation decomposition provides numerous problems including applications to hardware with fixed topology [5]. These aspects started to play critical role after first commercial quantum computing systems became available. Furthermore, hardware attacks, based on the security holes of conventional electronics,

Adam Glos

aglos@iitis.pl

1 Institute of Theoretical and Applied Informatics, Polish Academy of Sciences, Bałtycka 5, 44-100 Gliwice, Poland

2 Institute of Informatics, Silesian University of Technology, Akademicka 16, 44-100 Gliwice, Poland 
have been discovered for quantum cryptographic protocols [6]. The issues mentioned above demonstrated that the theoretical security confirmed by the laws of physics in the ideal environment could be deceptive in the real-world applications. For the users, it is important to be aware that quantum algorithms are inefficient for some types of input data. One of such examples is quantum spatial search, which is inefficient on 2D grid [1]. This demonstrates that the quantum algorithms are not only unsuitable for some types of problems, but, even for generally optimal quantum algorithms [7], it is possible to construct input data rendering them slow.

These examples show that, while quantum algorithms may be a milestone in computational theory, their practical implementation and security still need to be carefully checked. From the computational complexity point of view, it remains unknown whether the existing quantum algorithms can provide the speed-up promised by the theoretical results, especially when applied to large datasets. On the other hand, the ability to describe the types of data which render quantum algorithms ineffective can be used to develop post-quantum protocols.

Furthermore, as the remote utilization of quantum computational resources plays vital role for the development of quantum technologies, one should consider various aspects of the functioning of quantum devices accessed remotely. Currently, quantum transmission protocols used for Universal Blind Quantum Computation [8] can be used for transferring quantum data unknown to the server. Such protocols provide also correctness of the data and authentication of the transmission. However, they cannot be used for preventing the situation when maliciously prepared data are provided and result in the undesired behaviour of quantum machine. This might lead, for example, to denial-of-service effect. The analysis of the impact that the malformed data can have on the quantum computing infrastructure is still missing, and this aspect of using quantum computers in the cloud deserves more attention.

\subsection{Contribution}

In this paper, how the input data can influence the efficiency of quantum algorithms based on quantum spatial search is studied. We demonstrate that the efficiency of quantum algorithms can be significantly diminished by manipulation of the input data. For the purpose of this paper, we consider the following scenario. We have two parties, Alice and Bob. Alice prepares input data and sends them to Bob, who has for his disposal quantum computational resources. Alice requests from Bob some kind of processing, e.g., sorting a list or finding its maximum. This situation naturally occurs in cloud computing scenario. If the input data are altered by a third party, Eve, then the computational process executed by Bob may require more resources then initially assumed. In this situation, Bob might not be able to handle queries from other users. However, the client-server scenario we consider here can also include the possibility that Alice is responsible for sending data which are already malformed. This might result either from the purposeful acting of Alice or from the fact that the data contain instances of problems which are hard for quantum computers to handle.

As an example, one can consider a sorting via classical algorithm QuickSort. The algorithm achieves average-case performance $O(n \log (n))$, for number of elements $n$. 
However, for simple fixed pivot choice, one can provide malicious input data, which increase computation time up to $O\left(n^{2}\right)$. If no security is considered, choosing such data may considerably increase the computational resources required for running the algorithm. If the computer processes such data, this may result in denial-of-service. Fortunately, there are known solutions to this problem, for example, choosing pivot randomly.

Similar problems arise in the quantum spatial search, based on different models of quantum walks. For the continuous-time quantum walk, the algorithm works very well and securely on many graphs [7,9,10], achieving efficiency $O(\sqrt{n})$, for the graph order $n$. However, there are known examples, such as a two-dimensional grid, for which the algorithm reaches linear complexity only [1]. While the grid graph can be searched much faster for the discrete coined quantum walk, it is known that specific subgraphs of the searched graphs can form the so-called exceptional configurations [11] which strongly reduce the efficiency.

In this paper, we consider the formation of exceptional configurations as a method of attacking quantum spatial search. We propose a framework enabling the analysis of the attack efficiency based on the expected runtime of the algorithm. We utilize this framework for selected families of random graphs. We take into account different resources available for the attacker. We also discuss the connection between our results and the security protocols used for Universal Blind Quantum Computation.

\section{Preliminaries}

Let $G=(V, E)$ be an undirected graph and let $S \subset V$ be a set of searched vertices. A quantum spatial search algorithm is a quantum walk, which after $t$ steps finds any marked $v \in \mathrm{S}$ with probability $p$. While the classical spatial search is known to have time complexity $\Omega(n)$, it is possible to achieve $\Theta(\sqrt{n})$ for quantum algorithms $[1$, $7,10,12]$. The complexity may depend on a chosen graph [1] or a chosen set $S$ [13]. Throughout this paper, we assume that the complexity of the quantum spatial search grows as $\Theta\left(n^{\alpha}\right)$ for some $\alpha>0$ which is commonly observed for many types of graphs.

For discrete coined quantum walk (DCQW), the search problem with multiple marked vertices can be a hard task for some combinations of marked vertices, known as exceptional configurations $[11,13,14]$. The existence of an exceptional configuration is demonstrated in two steps. First, the existence of a special stationary state needs to be shown. Second, a bound on the probability needs to be determined, based on the stationary state. Recently, the class of connected subgraphs having the stationary state has been described, which solves the first step.

Theorem [15] Let $G$ be an arbitrary graph. Let $H$ be its induced connected subgraph. $H$ contains a stationary state, if

- it is not bipartite, or

- it is $\left(V_{H}^{1}, V_{H}^{2}\right)$-bipartite satisfying 


$$
\sum_{v \in V_{H}^{1}} \operatorname{deg}_{G}(v)=\sum_{v \in V_{H}^{2}} \operatorname{deg}_{G}(v)
$$

where $\operatorname{deg}_{G}$ is the degree in graph $G$.

The exceptional configuration of order 2 (2EC) is a path of length 2 , such that the degrees of vertices in the original graph $G$ are equal. The exceptional configuration of order 3 (3EC) is a triangle graph, or a path of length 3, such that the degree of the middle vertex equals the sum of the degrees of the end vertices in the original graph $G$. Wong has shown the equivalence between coined and Szegedy model (SzQW) $[16,17]$, which is a general quantum walk definable on an arbitrary directed graph. We are going to focus on the latter, as it is more suitable for numerical analysis.

\section{Description of the attack}

Let us assume that Alice sends to Bob a description of the oracle, for example in the form of the quantum circuit. Bob executes Szegedy quantum search based on the obtained oracle. As a result, Bob sends to Alice a marked vertex, found by the algorithm. In this situation, Bob expects to take advantage of the capabilities of quantum computer and obtain the result within time $\Theta\left(t^{\alpha}\right)$.

Let us now assume that Eve has her own quantum resources and intercepts the description of the oracle send by Alice. She executes the algorithm and finds the vertex in time $\Theta\left(t^{\alpha}\right)$. Then she creates a description of new oracle, in which additional vertices is marked, and sends it to Bob. If marked vertices form an exceptional configuration, then Bob will need $\Theta\left(t^{\beta}\right)$ time to execute the algorithm, with $\beta>\alpha$. One should note that modification of input data send by Eve cannot be corrected by using the error correction code, as the modification are made on a logical level.

Bob may detect that the oracle was altered if the algorithm consumes more resources that it was initially assumed. Additionally, we assume that Bob knows that the oracle has been modified by marking the additional vertices. This is the worst possible scenario from Eve's perspective. In this situation, he can modify the hyperparameters of his algorithm to limit the adverse effect. The simplest strategy is to change the measurement time. By this he may reduce the execution time to $\Theta\left(t^{\bar{\beta}}\right)$, with $\bar{\beta}<\beta$.

\subsection{Framework for quantifying the efficiency of attacks}

For the purpose of quantifying the efficiency of attacking methods, we introduce the formal description of the family of quantum spatial search algorithms.

Definition Quantum Spatial Search $\mathcal{Q S S}$ is a tuple (Alg, $t ; G, \mathbf{S}, \theta)$, where $\mathbf{A l g}$ is a quantum algorithm searching for any vertex $v \in \mathrm{S}$ in time $t$, running on graph $G$, and parametrized by the set of parameters $\theta$.

By $p(\mathcal{Q S S})$ we denote the success probability of $\mathcal{Q S S}$. Note that we do not define $\theta$ precisely, as it depends on the chosen algorithm Alg. For example, for coined quantum 
walk Alg = DCQW, the parametrization consists of the set of coin operators. For $\mathbf{A l g}=\mathrm{SzQW}$, the parametrization is the chosen stochastic operation $P$.

In order to compare different quantum spatial search algorithms, we propose the following measure of efficiency.

Definition Expected runtime $\mathcal{T}_{\mathcal{Q S S}}$ of quantum spatial search $\mathcal{Q S S}=(\mathbf{A l g}, t ; G, \mathrm{~S}, \theta)$ is defined as

$$
\mathcal{T}_{\mathcal{Q S S}}:=\frac{t}{p(\mathcal{Q S S})} .
$$

The expected runtime is an expectation of number of steps after which we get the result using Bernoulli process. Such approach has been used in [1,12], where the complexity was analysed.

Using the formalized description of quantum spatial search algorithms, we can introduce the concept of attack on an algorithm as follows.

Definition Attack on $\mathcal{Q S S}$ is a function $h$ such that

$$
h(\mathbf{A l g}, t ; G, \mathbf{S}, \theta)=\left(\mathbf{A l g}, t ; G^{\prime}, \mathbf{S}^{\prime}, \theta^{\prime}\right)
$$

It should be stressed that the attack cannot change the evolution model and measure time of $\mathcal{Q S S}$. Still we will consider the function altering only some of the elements of $\mathcal{Q S S}$. For example, the attacks restricted to graph structure imply that $\mathrm{S}=\mathrm{S}^{\prime}$ and $\theta=\theta^{\prime}$.

If we allow $\mathcal{Q S S}$ element to be changed, then we will say the element is hackable. Otherwise, it is not hackable. Note that by definition Alg and $t$ are not hackable.

To quantify the efficiency of the attacks, we introduce attack efficiency as follows.

Definition Attack efficiency eff ${ }_{h, \mathcal{Q S S}}$ on $\mathcal{Q S S}$ is defined as

$$
\operatorname{eff}_{h, \mathcal{Q S S}}:=1-\frac{\mathcal{T}_{\mathcal{Q S S}}}{\mathcal{T}_{h(\mathcal{Q S S})}}
$$

We are interested in such functions $h$ that $\operatorname{eff}_{h, \mathcal{Q S S}} \geq 0$. Furthermore, since $t$ is common for both $\mathcal{Q S S}$ and $h(\mathcal{Q S S})$ we have

$$
\operatorname{eff}_{h, \mathcal{Q S S}}=1-\frac{p(h(\mathcal{Q S S}))}{p(\mathcal{Q S S})}
$$

Let us consider the following scenario. The user is trying to start an algorithm $\mathcal{Q S S}$, but the attacker has changed it into $\mathcal{Q S S} \mathcal{S}^{\prime}$. If the user is aware of the attack and its nature, he can alter the measurement time $t$ in order to minimize the expected runtime. To describe this situation we introduce strong attack efficiency.

Definition Suppose we have $\mathcal{Q S S}=(\mathbf{A l g}, t ; G, \mathrm{~S}, \theta)$ and $h: \mathcal{Q S S} \mapsto\left(\mathbf{A l g}, t ; G^{\prime}, \mathbf{S}^{\prime}\right.$, $\left.\theta^{\prime}\right)$. Strong attack efficiency $\overline{\operatorname{eff}}_{h, \mathcal{Q S S}}$ is defined as

$$
\overline{\operatorname{eff}}_{h, \mathcal{Q S S}}:=1-\frac{\mathcal{T}_{(\mathbf{A l g}, t ; G, \mathrm{~S}, \theta)}}{\min _{\tau \geq 0} \mathcal{T}_{\left(\mathbf{A l g}, \tau ; G^{\prime}, \mathrm{S}^{\prime}, \theta^{\prime}\right)}} .
$$


This definition captures the best possible defence against the attack.

All of the above definitions were restricted to the fixed spatial search algorithm. Since our aim is to analyse the efficiency of attacks on more general graph classes, we extend the previously defined terms.

Definition Let $Q$ be a set of quantum spatial searches and $h$ be an attack on $Q$. Then maximal attack efficiency on $Q$, $\operatorname{eff}_{h, Q}$, is defined as

$$
\operatorname{eff}_{h, Q}:=\max _{\mathcal{Q S S} \in Q} \operatorname{eff}_{h, \mathcal{Q S S}}
$$

Similarly, maximal strong attack efficiency on $Q$, eff ${ }_{h, Q}$, is defined as

$$
\overline{\operatorname{eff}}_{h, Q}:=\max _{\mathcal{Q S} \mathcal{S} \in Q} \overline{\operatorname{eff}}_{h, \mathcal{Q S} \mathcal{S} .}
$$

The above definition captures the pessimistic level of robustness against the attack. Similarly, we can define the mean and minimal efficiencies. In practice, we would like to find the dependence between the efficiency and the order of the graph.

Instead of providing deterministic $Q_{n}$, we will focus on $Q_{n}$ defined by random graph models $\mathcal{G}_{n}$.

Definition Let $\mathcal{G}_{n}$ be a random graph model, and let for arbitrary $G$ exist a quantum spatial search $\mathcal{Q S S}(G)=(\mathbf{A l g}, t(G) ; G, \mathrm{~S}(G), \theta(G))$. Let $h$ be an attack defined on $\mathcal{Q S S}$. We say that the efficiency of the attack is almost surely at least $E_{n}$ iff

$$
\mathbb{P}\left(\operatorname{eff}_{h, \mathcal{Q S S}(G)} \geq E_{n} \mid G \in \mathcal{G}_{n}\right) \stackrel{n \rightarrow \infty}{\longrightarrow} 1
$$

The above definition can be naturally applied to strong efficiency.

\section{Attacks on quantum spatial search algorithms}

We consider the formation of exceptional configurations as a method of attacking quantum spatial search.

We will consider the attacks altering the set of marked vertices only. For quantum spatial search (SzQW, $t ; G,\{v\}, P_{u}$ ), we add marked vertices in such a way that the newly generated set $S^{\prime} \supset\{v\}$ forms a connected exceptional configuration. As the probability of finding any vertex from EC is much lower than the probability of finding a single marked vertex [11], we decrease the success probability of the algorithm.

Suppose that the algorithm outputs a vertex from $S^{\prime} \backslash\{v\}$. Since we choose additional marked vertices from close neighbourhood of the original marked vertex $v$, the possible defence against the attack is to make a simple classical search over its neighbourhood, which is efficient for sparse graphs. However, the cumulated success probability of measuring any of $S^{\prime}$ is small. Hence, the attack is effective.

Let us analyse Erdôs-Rényi, Watts-Strogatz, and Barabási-Albert models. For Erdôs-Rényi, we choose the probability of adding edge $p=\frac{2 \log (n)}{n}$, for WattsStrogatz we select initial degree $K=\lceil 2 \log n\rceil$ and randomness $\beta=0.5$ [18], and for 


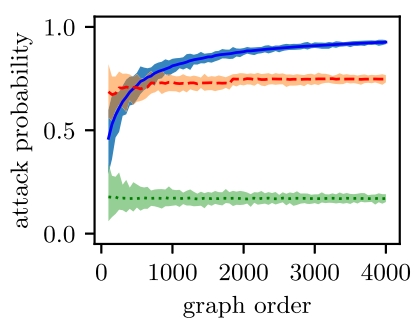

(a) EC of order 2

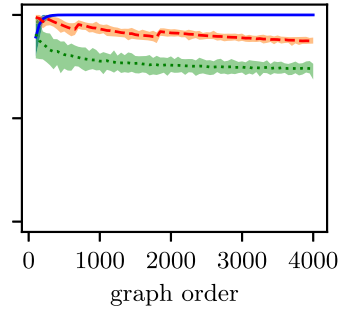

(b) $\mathrm{EC}$ of order 2 or 3

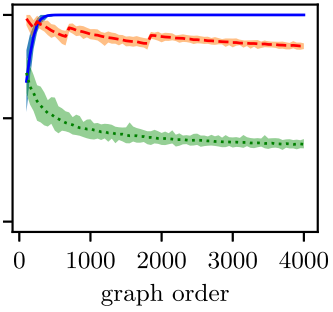

(c) EC of order 2 or 3 within distance 1

Fig. 1 Probability of forming an exceptional configuration with a random vertex for different models of random graphs. a Probability of forming EC of order 2; b probability of forming EC of order 2 or 3; c probability of forming EC of order 2 or 3 within distance 1 from initially marked. For each graph order $n=100,150, \ldots, 4000$, we have analysed 100 graphs. The area describes the range of attack probability obtained from sampled graphs. Blue solid lines describe Erdős-Rényi graphs, orange dashed lines describe Watts-Strogatz graphs, and green dotted lines describe Barabási-Albert graphs (Color figure online)

Barabási-Albert we set attachment parameter to $m_{0}=3[19,20]$. First, we analyse the probability that for randomly chosen vertex $v$ we can construct an exceptional configuration $S$ such that $v \in S$. Next, we analyse the efficiency of the attack.

Numerical results demonstrate that for every vertex of Watts-Strogatz graph we can almost surely find an exceptional configuration of order 3 (3EC), see Fig. 1. Thus the model can be attacked almost surely for any vertex. This is no longer the case when we allow constructing an exceptional configuration of order 2 only. For Erdős-Rényi and Barabási-Albert models, even 3EC will not provide this kind of advantage for the attacker. Nevertheless, the probability of attacking is still large, and the attacker may be able to construct an exceptional configuration of a higher order.

Another constraint for resources available to the attacker is the distance between the originally marked vertices and the newly marked ones. We consider two scenarios. For local exceptional configurations, the new vertices are direct neighbours of the original vertex. In the global scenario, they are at most neighbours of neighbours. Based on Fig. 1, one can see that it is much easier to find global exceptional configurations than the local ones for Barabási-Albert model. Hence the probability of attacking increases if the attacked has the ability to use such EC. For Erdős-Rényi model, the attacker cannot utilize this type of advantage, for given parametrization. Furthermore, for $p=\omega(\log (n) / n)$ all vertices have almost equal degrees [21]. This makes finding path graph 3EC impossible, as degree condition from Preliminaries section cannot be fulfilled. We have not observed any dependence for Watts-Strogatz model.

For analysing the efficiency of the attack, we have chosen at random two vertices forming an exceptional configuration. We have analysed 50 graphs for each order $n=100,150, \ldots, 2400$ for all models, using the optimization algorithm implemented in QUANTUMWALK package ${ }^{1}$ with penalty time $t_{\text {pen }}=\lceil\log (n)\rceil$. Parameter $t_{\text {pen }}$ is a value which is added to the time, thus changing the expected time into $\left(t+t_{\text {pen }}\right) / p$. Such adjustment prevents the optimization algorithm from halting at small time. Since $\log (n)$ is typically much smaller than $t$, its impact on our results is negligible.

\footnotetext{
1 Code available at https://github.com/iitis/QuantumWalk.jl.
} 

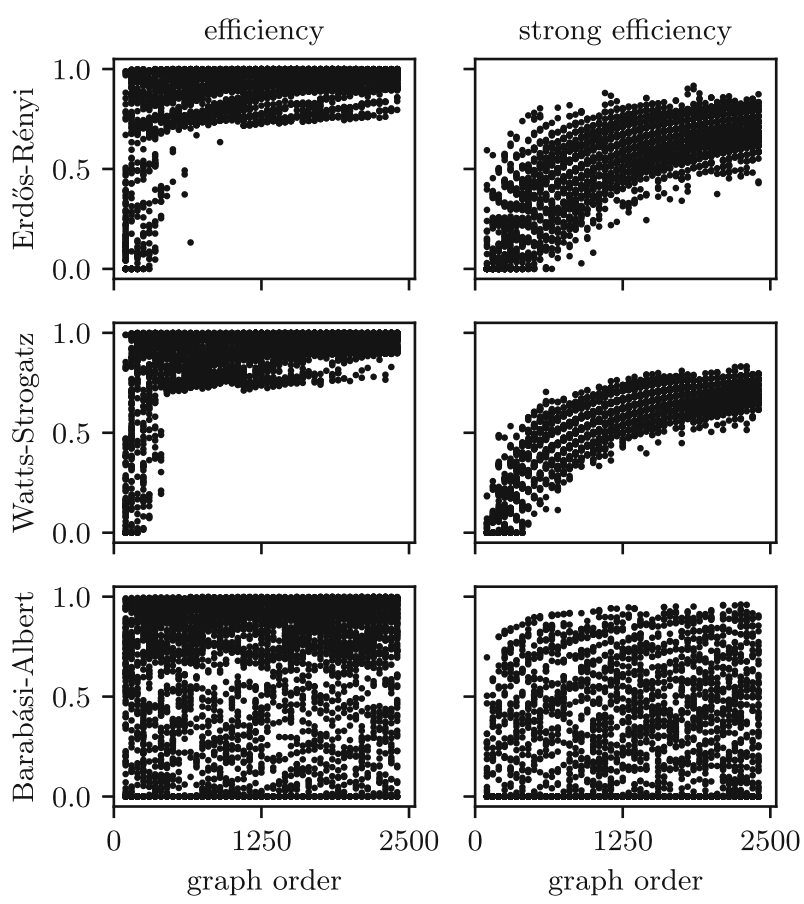

Fig. 2 Attack efficiency and strong attack efficiency for random graph models. Pairs of vertices were chosen at random from the collection of all 2EC. Left plots present the efficiency (the case of unchanged measurement time). Right plots present the strong efficiency (the case with measurement time chosen optimally). While Erdős-Rényi and Watts-Strogatz present similar results, one can observe more robust behaviour for Barabási-Albert model

In order to asses the impact of the attack on the complexity of quantum spatial search, we have calculated the expected runtime for three cases for each random graph. In the first case, which represents the reference search, we have a single marked element. This case provides the value of efficiency of the search $\mathcal{T}_{\mathcal{Q S} S}$ for a given graph. Next, we execute the $\mathcal{Q S S}$ on the graph modified by the addition of a single marked element, which results in the formation of an exceptional configuration. This case provides us the value of the increase of the expected runtime resulting from the attack and thus the efficiency of the attack [cf. Eq. (4)]. Finally, we calculate the expected runtime for the case when the user is aware of the attack and can utilize this knowledge to minimize its effects. In this case, we calculate the strong efficiency (cf. Eq. 6).

Numerical results presented in Fig. 2 show that the attacking efficiency depends on the chosen graph model. Left three plots show that if user is not aware of the attack, the efficiency is almost surely 0.8 for Erdôs-Rényi and almost surely 0.9 for Watts-Strogatz. For Barabási-Albert, the results are much more irregular, and no level of efficiency can be guaranteed. This suggests there are some other parameters influencing the efficiency of the attack.

If the user is aware of the attack, it is possible to prevent it at least partially for all models by changing the measurement time. The efficiency in this case is captured 

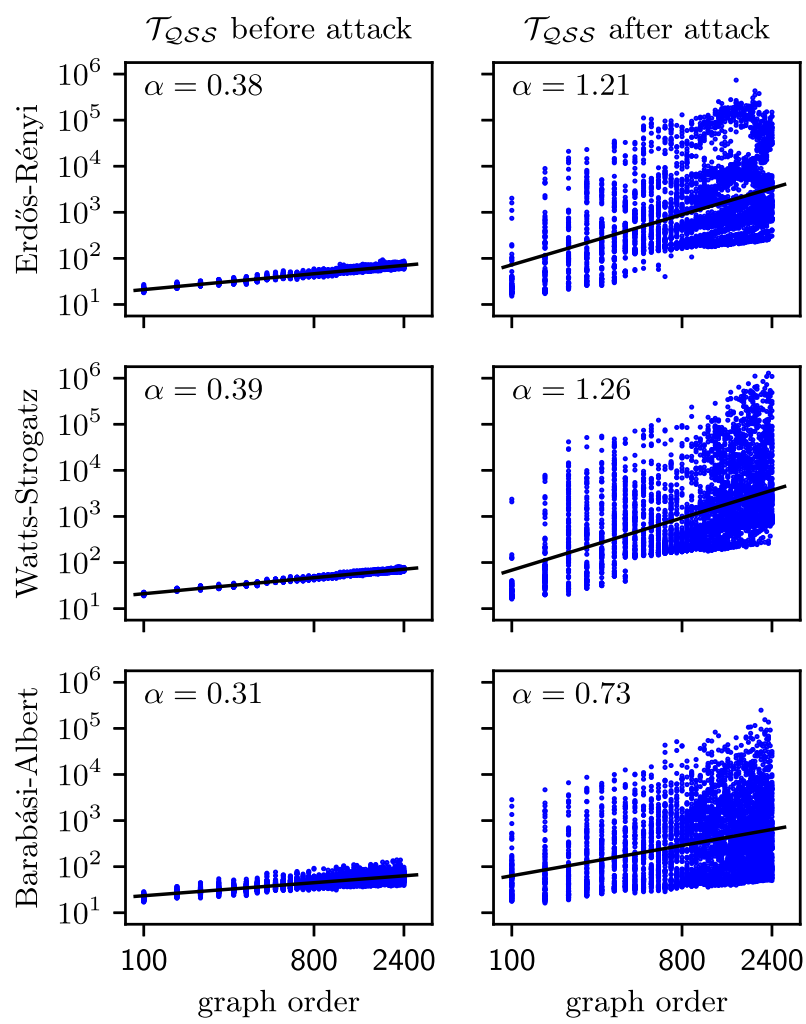

Fig. 3 Comparison of efficiency for the reference search (left plots) and the search after the attack (right plots) for different models of random graphs. Right plots provide detailed analysis of results concerning the efficiency case from Fig. 2. The tangent of regression line, denoted by $\alpha$, is the numerically derived complexity $\Theta\left(n^{\alpha}\right)$. One can observe the increase in the runtime resulting from the attack for Erdős-Rényi and Watts-Strogatz models

by the notion of strong efficiency. For Erdős-Rényi and Watts-Strogatz models, the strong efficiency is almost surely at least 0.5. For Barabási-Albert, we still observe high irregularity in obtained data and the strong efficiency is almost surely significantly smaller than 1 .

As the complexity attack should result in the algorithm complexity growth, we have determined numerically the expected time change in the case of common measurement time. The results are presented in Fig. 3. We have assumed that the complexity grows as the power complexity, $\Theta\left(n^{\alpha}\right)$. The tangent of the regression line of the expected runtime in the function of the graph order on the log-log scale provides the approximation of the parameter $\alpha$. The observed growth of the values of $\alpha$, resulting from the attack, demonstrates that the attacker is able to significantly increase the expected runtime of the quantum algorithm. This suggests that the algorithm is vulnerable to the complexity attack, which might result in the denial-of-service of the quantum computer. 


\section{Summary and discussion}

In this paper, we have signified the problem of possible vulnerability of quantum algorithms to the complexity attacks. The presented approach is based on the analysis of input data. As such it can be used to discover weaknesses of quantum computers resulting from the application of quantum algorithms on input data unsuitable for processing on quantum machines. This is in contrast to the common approach where only the theoretical computational complexity is taken into account.

We have developed the theoretical framework for quantifying the efficiency of the attacks. We have constructed an attack based on exceptional configurations and analysed it in the context of its applicability and efficiency. The analysis confirms that it is possible to decrease the efficiency of quantum spatial search based on Szegedy walk by malicious modification of input data.

One should note that the presented results can be applied for a general class of graphs. This is in contrast to the results from [11], where only special classes of graphs were considered. For those classes, it can be shown analytically that the algorithm complexity changes from $\Theta(\sqrt{n})$ to $\Theta(n)$.

It should be stressed that the models of random graphs used for assessing the security of quantum algorithms mimic the structure of real-world data [20]. As such the presented analysis confirms that the theoretical security of quantum procedures can be inadequate when the algorithms are applied for specific input data. This includes input data which encode the connections observed in complex networks.

We should also stress that the scenario considered in this work does not focus on the security of the communication which takes place between the client and the server. On the contrary, our figure of merit is the impact of the modified data on the computational resource used by the server. This is in contrast to the protocols based on the concept of Universal Blind Quantum Computation [8]. Such protocols enable the detection of the input data modification, if the data are encoded in a quantum state. Alice can use such protocols to counteract the effect of data alteration by informing Bob about the Eve's activity. However, such protocol cannot be used to prevent Alice from sending malformed input data. On the other hand in our scenario it is easy to include the possibility that Alice is responsible for sending data which are already malformed-either on purpose or by accident-and our goal is to asses how often such situation can occur when we consider data modelled by complex networks. Our results show that secure transmission is insufficient for ensuring the availability of the quantum hardware.

Acknowledgements The authors would like to thank Nikolay Nahimov for discussion concerning exceptional configurations and Alexander Rivosh for inspiring the application of this concept in quantum spatial search, and Iza Miszczak for reviewing the manuscript. This work has been partially supported by Polish National Science Center Grant 2011/03/D/ST6/00413. Numerical calculations were possible thanks to the support of PL-Grid Infrastructure. We would also like to thank the anonymous reviewer for his comments, which greatly improved the quality of our paper.

Open Access This article is distributed under the terms of the Creative Commons Attribution 4.0 International License (http://creativecommons.org/licenses/by/4.0/), which permits unrestricted use, distribution, and reproduction in any medium, provided you give appropriate credit to the original author(s) and the source, provide a link to the Creative Commons license, and indicate if changes were made. 


\section{References}

1. Childs, A.M., Goldstone, J.: Spatial search by quantum walk. Phys. Rev. A 70(2), 022314 (2004)

2. Harrow, A.W., Montanaro, A.: Quantum computational supremacy. Nature 549(7671), 203-209 (2017)

3. Bernstein, D.J., Lange, T.: Post-quantum cryptography. Nature 549(7671), 188 (2017)

4. Van Meter, R., Horsman, C.: A blueprint for building a quantum computer. Commun. ACM 56(10), 84-93 (2013)

5. Saeedi, M., Wille, R., Drechsler, R.: Synthesis of quantum circuits for linear nearest neighbor architectures. Quantum Inf. Process. 10, 355-377 (2011)

6. Lydersen, L., Wiechers, C., Wittmann, C., Elser, D., Skaar, J., Makarov, V.: Hacking commercial quantum cryptography systems by tailored bright illumination. Nat. Photonics 4(10), 686-689 (2010)

7. Chakraborty, S., Novo, L., Ambainis, A., Omar, Y.: Spatial search by quantum walk is optimal for almost all graphs. Phys. Rev. Lett. 116(10), 100501 (2016)

8. Broadbent, A., Fitzsimons, J., Kashefi, E.: Universal blind quantum computation. In: 2009 50th Annual IEEE Symposium on Foundations of Computer Science. IEEE, pp. 517-526 (2009)

9. Chakraborty, S., Novo, L., Di Giorgio, S., Omar, Y.: Optimal quantum spatial search on random temporal networks. Phys. Rev. Lett. 119, 220503 (2017)

10. Glos, A., Krawiec, A., Kukulski, R., Puchała, Z.: Vertices cannot be hidden from quantum spatial search for almost all random graphs. Quantum Inf. Process. 17, 81 (2018)

11. Nahimovs, N., Santos, R.A.: Adjacent vertices can be hard to find by quantum walks. In: SOFSEM 2017: SOFSEM 2017: Theory and Practice of Computer Science, LNCS, vol. 10139, pp. 256-267 (2017)

12. Ambainis, A., Kempe, J., Rivosh, A.: Coins make quantum walks faster. In: Proceedings of the 16th ACM-SIAM SODA, pp. 1099-1108 (2005)

13. Nahimovs, N., Rivosh, A.: Exceptional configurations of quantum walks with Grover's coin. In: International Doctoral Workshop on Mathematical and Engineering Methods in Computer Science. Springer, pp. 79-92 (2015)

14. Khadiev, K., Nahimovs, N., Santos, R.: On the probability of finding marked connected components using quantum walks. Lobachevskii J. Math. 39(7), 1016-1023 (2018)

15. Prūsis, K., Vihrovs, J., Wong, T.G.: Stationary states in quantum walk search. Phys. Rev. A 94(3), 032334 (2016)

16. Szegedy, M.: Quantum speed-up of Markov chain based algorithms. In: Proceedings of the FOCS 2004. IEEE, pp. 32-41 (2004)

17. Wong, T.G.: Equivalence of Szegedy's and coined quantum walks. Quantum Inf. Process. 16, 215 (2017)

18. Watts, D.J., Strogatz, S.H.: Collective dynamics of 'small-world' networks. Nature 393(6684), 440 (1998)

19. Barabási, A.-L., Albert, R.: Emergence of scaling in random networks. Science 286(5439), 509-512 (1999)

20. Albert, R., Barabási, A.-L.: Statistical mechanics of complex networks. Rev. Mod. Phys. 74(1), 47 (2002)

21. Bollobás, B.: Cambridge Studies in Advanced Mathematics. Modern Graph Theory, 2nd edn. Cambridge University Press, Cambridge (2001)

Publisher's Note Springer Nature remains neutral with regard to jurisdictional claims in published maps and institutional affiliations. 